\title{
Causality and Collisionless Damping in Plasma
}

\author{
Hee J. Le ${ }^{1 *}$, Mi Young Song ${ }^{2}$ \\ ${ }^{1}$ Department of Physics, Hanyang University, Seoul, Korea \\ ${ }^{2}$ Plasma Technology Research Center, National Fusion Research Institute, Gunsan, Korea \\ Email: *ychjlee@yahoo.com
}

Received January 21, 2013; revised February 22, 2013; accepted March 2, 2013

Copyright (C) 2013 Hee J. Lee, Mi Young Song. This is an open access article distributed under the Creative Commons Attribution License, which permits unrestricted use, distribution, and reproduction in any medium, provided the original work is properly cited.

\begin{abstract}
We derive the collisionless Landau damping in a plasma by satisfying the causal requirement that the susceptibility function of the plasma for time $t<0$ should be nil. The causality condition should be satisfied by the susceptibility function of a plasma no matter what equations we employ to describe the plasma. Thus we conclude that the fundamental reason of the collisionless damping can be traced to the causality. As an example, we derive the collisionless damping of ion acoustic wave in a plasma by employing fluid equations.
\end{abstract}

Keywords: Causality; Landau Damping; Ion Acoustic Wave

\section{Introduction}

Landau damping discovered by L. Landau in his famous paper [1] is collisionless damping, i.e. damping irrelevant to particle-particle collisions in plasmas. Landau solved the Vlasov-Poisson equations as an initial value problem by means of Laplace transform. In the course of analysis, one has to deal with the singular integral in the expression of the susceptibility function

$$
\chi(k, \omega)=\frac{\omega_{p e}^{2}}{k} \int_{-\infty}^{\infty} \mathrm{d} v \frac{\mathrm{d} g_{0}}{\mathrm{~d} v} \frac{1}{\omega-k v}
$$

where $\omega_{p e}$ is the electron plasma frequency, $g_{0}(v)$ is the zero order equilibrium velocity distribution function. In the velocity integral in Equation (1), $\omega=\omega_{r}+i \omega_{i}$ is a complex frequency and $v$ is a real variable. If the imaginary frequency $\omega_{i}$ becomes zero, and thus $\omega$ lands on somewhere on the real $v$-axis, the velocity integral becomes undefined since the integrand is singular at $v=\omega / k$. Landau evaluated this singular integral along his celebrated Landau contour by invoking the mathematical argument of analytic continuation in the complex $\omega$-plane. The Landau contour for the integral of Equation (1) consists of the principal value part along the real $v$-axis plus the infinitesimal semi-circle around the singular point $v=\omega / k$ in a concave-down shape. Therefore, the value of the $v$-integral is the sum of the principal value and the value contributed by the infinitesimal semicircle around the singular point $v=\omega / k$ which can be

${ }^{*}$ Corresponding author. expressed in terms of $\delta$-function. Thus, Equation (1) yields the real part $\operatorname{Re} \chi(k, \omega)$ corresponding to the principal value of the integral as well as the imaginary part $\operatorname{Im} \chi(k, \omega)$ obtained from the $\delta$-function part. Depending upon the slope of the distribution function, $\operatorname{Im} \chi(k, \omega)$ can give rise to either damping or growing of the plasma wave.

In this work, we show that the Landau damping can be derived from the causal requirement that must be satisfied for the dielectric permittivity function. The causal requirement is mathematically expressed by Equation (3), and should be satisfied always in electrodynamics no matter what way it is derived. Its physical meaning is that the response of the medium must follow the cause; the cause cannot be precedent to the effect. By enforcing this causality condition, the velocity integral in Equation (1) is shown to be equivalent to the integral along the Landau contour.

Recently Lim and Lee [2] derived the Landau damping from the Kramers-Kronig relations, and concluded that the fundamental reason of the collisionless damping of plasma waves can be traced to the causality. In this work, we derive the collisionless damping directly from the causal requirement without the aid of the KramersKronig relations; thus, we show more directly that the collisionless damping is a consequence of the causality. It is emphasized that $\operatorname{Im} \chi(k, \omega)$ is derived without the mathematical argument of the analytic continuation. As an example, we employ the fluid equations to derive the collisionless damping of an ion acoustic wave; this deri- 
vation clearly shows that the causal requirement is responsible for the collisionless damping.

\section{Evaluation of Susceptibility from the Causal Requirement}

In evaluation of plasma wave dispersion relation, one often encounters with the algebraic expression, the reciprocal of the Doppler-shifted frequency, $\frac{1}{\omega-k v}$. This algebraic quantity is well defined if $\omega \neq k v$, but it should be more defined when $\omega=k v$ because the denominator gives a singularity. In this case, the expression should be rephrased so that the singularity can be dealt with without ambiguity. We can use:

$$
\frac{1}{\omega-k v}=P \frac{1}{\omega-k v}+\lambda \delta(\omega-k v)
$$

where the symbol $P$ denotes the principal value and $\lambda$ is an undetermined constant. Equation (2) is correct because of the two identities:

$$
(x-a) P \frac{1}{x-a}=1,(x-a) \delta(x-a)=0
$$

In fact, Equation (2) was the mathematical motivation of the Van Kampen modes of plasma wave [3]. Here we use the causal requirement in electrodynamics to determine the constant $\lambda$.

Causality in electrodynamics means that the response must always follow the cause; the cause cannot be precedent to the effect. Therefore, the future susceptibility should be irrelevant to the present field (displacement). This causal notion leads to the mathematical condition expressed by [4]

$$
\chi(t)=0 \text { for } t<0
$$

where $\chi$ is the susceptibility of the medium.

As an application, we consider the susceptibility of electron plasma (ions are assumed to be immobile) governed by the Vlasov-Poisson equation as given by Equation (1). Using Equation (2) in Equation (1), the susceptibility function takes the form

$$
\chi(k, \omega)=\frac{\omega_{p e}^{2}}{k} \int_{-\infty}^{\infty} \mathrm{d} v \frac{\mathrm{d} g_{0}}{\mathrm{~d} v}\left[P \frac{1}{\omega-k v}+\lambda \delta(\omega-k v)\right]
$$

In order to determine the constant $\lambda$, let us invert Equation (4):

$$
\begin{aligned}
\chi(k, t) & =\int_{-\infty}^{\infty} \chi(k, \omega) \mathrm{e}^{-\mathrm{i} \omega t} \frac{\mathrm{d} \omega}{2 \pi} \\
& =\frac{\omega_{p e}^{2}}{2 \pi k} \int_{-\infty}^{\infty} \mathrm{d} v \frac{\mathrm{d} g_{0}}{\mathrm{~d} v} \mathrm{e}^{-\mathrm{i} k v t}\left[P \int_{-\infty}^{\infty} \mathrm{d} \omega \frac{\mathrm{e}^{-\mathrm{i} \omega t}}{\omega}+\lambda\right]
\end{aligned}
$$

The principal part integral is the step function $[3,5]$ :

$$
P \int_{-\infty}^{\infty} \frac{\mathrm{e}^{-\mathrm{i} x t}}{x} \mathrm{~d} x=\mathrm{i} \pi(\text { when } t<0) \text { or }-\mathrm{i} \pi(\text { when } t>0)
$$

The causal requirement in Equation (3) is satisfied by $\lambda=-\mathrm{i} \pi$, and therefore the plasma susceptibility in Equation (4) becomes

$$
\chi(k, \omega)=\frac{\omega_{p e}^{2}}{k} \int_{-\infty}^{\infty} \mathrm{d} v \frac{\mathrm{d} g_{0}}{\mathrm{~d} v}\left[P \frac{1}{\omega-k v}-\mathrm{i} \pi \delta(\omega-k v)\right]
$$

which agrees with the susceptibility evaluated along the Landau contour. Therefore we conclude that the collisionless damping of plasma waves is fundamentally due to the causal requirement.

\section{Ion Landau Damping Derived from Fluid Equations}

An ion in the electric wave of a Fourier component with phasor $\mathrm{e}^{\mathrm{i} k \mathrm{i}-\mathrm{i} t}$ is subject to the equation of motion

$$
\frac{\mathrm{d} \nu}{\mathrm{d} t}=\frac{e}{m_{i}} E(k, \omega) \mathrm{e}^{\mathrm{i}(k x-\omega t)}
$$

In Equation (8), the Lagrangian equation of motion, $x=x(t)$, the particle position at time $t$, and the sole independent variable is $t$. As the zero order solution, we use the unperturbed orbit

$$
x=x_{0}+v_{0} t
$$

where $x_{0}$ and $v_{0}$ are the initial position and velocity, respectively, at the initial time $t=0$. Then Equation (8) with the aforementioned boundary conditions is solved by

$$
v(t)=v_{0}-\frac{e}{m_{i}} E(k, \omega) \frac{\mathrm{e}^{\mathrm{i} k x_{0}}}{\mathrm{i}\left(k v_{0}-\omega\right)}\left[1-\mathrm{e}^{\mathrm{i}\left(k v_{0}-\omega\right) t}\right]
$$

The terms $v_{0}$ and 1 in the above equation are necessary to have the initial condition satisfied. However, even without those terms, the rest of the terms in Equation (9) satisfies Equation (8). Equation (9) is the particular solution of the differential Equation (8). The the first order homogeneous solution is meant by

$$
v(t)=\frac{e}{m_{i}} E(k, \omega) \frac{\mathrm{e}^{\mathrm{i} k x_{0}+\mathrm{i}\left(k v_{0}-\omega\right) t}}{\mathrm{i}\left(k v_{0}-\omega\right)}
$$

which corresponds to the Fourier component with phasor $\mathrm{e}^{\mathrm{i} k x-\mathrm{i} \omega t}$. It is trivial to obtain Eulerian velocity corresponding to Equation (10); we simply put $x_{0}=x-v_{0} t$ in Equation (10) to get

$$
v(x, t)=\frac{e}{m_{i}} E(k, \omega) \frac{\mathrm{e}^{\mathrm{i} k x-\mathrm{i} \omega t}}{\mathrm{i}\left(k v_{0}-\omega\right)}
$$

We immediately recognize that Equation (11) solves the Eulerian equation of motion 


$$
\frac{\partial v}{\partial t}+v_{0} \frac{\partial v}{\partial x}=\frac{e}{m_{i}} E(k, \omega) \mathrm{e}^{\mathrm{i} k x-\mathrm{i} \omega t}
$$

where $x$ and $t$ are now independent. The above Lagrangian consideration clarifies the meaning of $v_{0}$ which is the initial velocity, and $v$ is the perturbed velocity.

Proceeding from the above preliminary consideration of fluid equation, we derive the collisionless damping of ion acoustic wave via the fluid equations. We need both electron and ion equations. The plasma at hand is considered to be a group of ion beams; each beam is characterized by the initial velocity $v_{0}$ in the background of Boltzmann-distributed electrons. Modeling a plasma as a group of beams with varying beam velocities was earlier adopted by Bohm and Gross [6]. The ion equations read

$$
\begin{gathered}
\frac{\partial v}{\partial t}+v_{0} \frac{\partial v}{\partial x}=-\frac{e}{m_{i}} \nabla \phi \\
\frac{\partial n}{\partial t}+N\left(v_{0}\right) \frac{\partial v}{\partial x}+v_{0} \frac{\partial n}{\partial x}=0
\end{gathered}
$$

where $v_{0}$ is the zero order initial ion velocity, $v$ is the perturbed ion velocity, $N\left(v_{0}\right)$ is the equilibrium ion number density of the $v_{0}$-beam, $n$ is the perturbation of the ion number density of the $v_{0}$-beam, and $\phi$ is the electric potential $(\boldsymbol{E}=-\nabla \phi)$.

We assume that the electrons are Boltzmann-distributed in the background of the plasma. Thus, the perturbed electron number density in each beam can be written as

$$
n_{e}=N\left(\mathrm{e}^{\frac{e \phi}{T_{e}}}-1\right) \simeq \frac{e \phi}{T_{e}} N\left(v_{0}\right)
$$

The Poisson equation reads

$$
\nabla^{2} \phi=-4 \pi e \sum_{v_{0}}\left[n\left(v_{0}\right)-\frac{e \phi}{T_{e}} N\left(v_{0}\right)\right]
$$

Equations (13) and (14) yield, in terms of the Fourier amplitudes,

$$
n\left(k, \omega, v_{0}\right)=\frac{N\left(v_{0}\right)}{\left(\omega-k v_{0}\right)^{2}} \frac{e k^{2} \phi}{m_{i}}
$$

Substituting Equation (17) into the Fourier transformed equation of Equation (16) and integrating over the distribution of the initial velocities $f\left(v_{0}\right)$ by putting $N\left(v_{0}\right)=f\left(v_{0}\right) \mathrm{d} v_{0}$, we obtain the dispersion relation,

$$
\begin{aligned}
\varepsilon(k, \omega) & =1+\chi(k, \omega) \\
& =1+\frac{1}{k^{2} \lambda_{D}^{2}}-\omega_{p i}^{2} \int_{-\infty}^{\infty} \frac{f\left(v_{0}\right) \mathrm{d} v_{0}}{\left(\omega-k v_{0}\right)^{2}}=0
\end{aligned}
$$

where $\omega_{p i}$ is the ion plasma frequency and the Debye length of the plasma is defined by

$$
\lambda_{D}^{-2}=\frac{4 \pi e^{2}}{T_{e}} \int_{-\infty}^{\infty} f\left(v_{0}\right) \mathrm{d} v_{0}
$$

Integrating by parts in Equation (18), the susceptibility is found to be

$$
\chi(k, \omega)=\frac{1}{k^{2} \lambda_{D}^{2}}+\frac{\omega_{p i}^{2}}{k} \int_{-\infty}^{\infty} \mathrm{d} v_{0} \frac{\frac{\mathrm{d} f}{\mathrm{~d} v_{0}}}{\omega-k v_{0}}
$$

In order to enforce the causal requirement $\chi(k, t<0)=0$, we take the steps parallel to Equations (3)-(7) by introducing Equation (2) for the quantity $1 /\left(\omega-k v_{0}\right)$ in the above integral. We are led to the following equation by the causal requirement.

$$
\begin{aligned}
& \chi(k, \omega) \\
& =\frac{1}{k^{2} \lambda_{D}^{2}}+\frac{\omega_{p i}^{2}}{k} \int_{-\infty}^{\infty} \mathrm{d} v_{0} \frac{\mathrm{d} f}{\mathrm{~d} v_{0}}\left[P \frac{1}{\omega-k v_{0}}-\mathrm{i} \pi \delta\left(\omega-k v_{0}\right)\right]
\end{aligned}
$$

The first term on the right side of Equation (20) comes from the Boltzmann-distributed electron density which replaces the electron Vlasov equation. Equation (20) agrees with the kinetic theory result. In summary, we derived the the susceptibility of ion acoustic wave via fluid equations and without resorting to the argument of analytic continuation; we used more direct condition of the causal requirement expressed by Equation (3).

\section{Discussion}

We have shown that $\operatorname{Im} \chi(\omega)$ as obtained by the Landau contour follows from the causal requirement expressed by Equation (3), $\chi(t<0)=0$. This derivation of the collisionless damping makes it transparent that the causality is responsible for the collisionless damping. The collisionless damping of plasma waves appears to be universal because the causality prevails regardless of the way of describing plasmas.

The causal requirement, $\chi(t<0)=0$, is mathematically equivalent to the analyticity of $\chi(\omega)$ in the upper half $\omega$-plane; this analyticity is basic to the derivation of the Landau damping via the argument of the analytic continuation, as is read in standard text books. It can be also shown that the causality condition directly leads to the Kramers-Kronig relations [2].

We also showed that Landau damping can also be derived from the fluid equations by applying the causal requirement. Understanding the Landau damping in terms of causality might provide further insight for plasma electrodynamics.

The Cerenkov radiation is emitted by a medium and is known to be the inverse process of Landau damping. The Cerenkov radiation may be also interpreted in the light of causality. 


\section{Acknowledgements}

The authors thank Dr. Y. D. Jung at Hanyang University for many valuable discussions.

\section{REFERENCES}

[1] L. Landau, "On the Vibrations of the Electronic Plasma," Journal of Physics, Vol. 10, No. 1, 1946, pp. 25-34.

[2] Y. K. Lim and H. J. Lee, "Causality, Kramers-Kronig Relations, and Landau Damping," The Open Plasma Physics Journal, Vol. 5, No. 1, 2012, pp. 36-40.
[3] N. G. Van Kampen and B. U. Felderhof, "Theoretical Methods in Plasma Physics," John Wiley, New York, 1967.

[4] J. D. Jackson, "Classical Electrodynamics," Wiley, New York, 1974

[5] G. Arfken, "Mathematical Methods for Physicists," Academic Press, Orlando, 1985, p. 415.

[6] D. Bohm and E. P. Gross, "Theory of Plasma Oscillations. A. Origin of Medium-Like Behavior," Physical Review, Vol. 75, No. 12, 1949, pp. 1851-1864. 